Consensus Statement

\title{
Current practice of pressurized intraperitoneal aerosol chemotherapy (PIPAC): Still standardized or on the verge of diversification?
}

\author{
Olivia Sgarbura ${ }^{a}{ }^{*}$, Laurent Villeneuve ${ }^{b}$, Mohammad Alyami ${ }^{\text {c }}$, Naoual Bakrin ${ }^{\mathrm{d}}$, \\ Juan José Torrent ${ }^{\mathrm{e}}$, Clarisse Eveno ${ }^{\mathrm{f}}$, Martin Hübner ${ }^{\mathrm{g}}$, on the behalf of the \\ ISSPP PIPAC study group \\ a Department of Surgical Oncology, Montpellier Cancer Institute, University of Montpellier, France \\ b Department of Clinical and Epidemiological Research, Department of Public Health, Lyon Sud University Hospital, EA, 3738, Lyon 1 University, Lyon, France \\ c Department of Surgery, Oncology center, King Khalid hospital, Najran, Saudi Arabia \\ ${ }^{\mathrm{d}}$ Department of Digestive Surgery, Lyon Sud University Hospital, EA, 3738, Lyon 1 University, Lyon, France \\ ${ }^{\mathrm{e}}$ Department of Surgical Oncology, Torrent Quénet Institute, Barcelona, Spain \\ ${ }^{\mathrm{f}}$ Department of Digestive and Oncologic Surgery, Claude Huriez University Hospital, Centre Hospitalier Universitaire (CHU), Lille \\ ${ }^{g}$ Department of Visceral Surgery, Lausanne University Hospital CHUV, University of Lausanne (UNIL), Switzerland
}

\section{A R T I C L E I N F O}

\section{Article history:}

Received 10 May 2020

Accepted 20 August 2020

Available online 29 August 2020

\section{Keywords:}

ePIPAC

Technique

Treatment protocol

Indications

Safety

Peritoneal metastasis

PRGS

\begin{abstract}
A B S T R A C T
Background: PIPAC is a new treatment modality for peritoneal cancer which has been practiced and evaluated until very recently by few academic centers in a highly standardized manner. Encouraging oncological outcomes and the safety profile have led to widespread adoption. The aim of this study was to assess current PIPAC practice in terms of technique, treatment and safety protocol, and indications. Methods: A standardized survey with 82 closed-ended questions was sent online to active PIPAC centers which were identified by help of PIPAC training centers and the regional distributors of the PIPAC-specific nebulizer. The survey inquired about center demographics $(n=8)$, technique $(n=34)$, treatment and safety protocol $(\mathrm{n}=34)$, and indications $(\mathrm{n}=6)$.

Results: Overall, 62 out of 66 contacted PIPAC centers answered the survey (response rate 93\%). 27 centers had performed $>60$ PIPAC procedures. A consensus higher than $70 \%$ was reached for 37 items (50\%), and higher than $80 \%$ for 28 items (37.8\%). The topics with the highest degree of consensus were safety and installation issues (93.5\% and $80.65 \%$ ) while chemotherapy and response evaluation were the least consensual topics ( 63.7 and 59.6\%). The attitudes were not influenced by volume, PIPAC starting year, type of activity, or presence of peritoneal metastases program.

Conclusion: Homogeneous treatment standards of new techniques are important to guarantee safe implementation and practice but also to allow comparison between cohorts and multi-center analysis of merged data including registries. Efforts to avoid diversification of PIPAC practice include regular update of the PIPAC training curriculum, targeted research and a consensus statement.
\end{abstract}

๑ 2020 Elsevier Ltd, BASO The Association for Cancer Surgery, and the European Society of Surgical Oncology. All rights reserved.
Abbreviations: ePIPAC, electrostatic Pressurized intraperitoneal aerosol chemotherapy; HIPEC, Hyperthermic intraperitoneal chemotherapy; ISSPP, International Society for the Study of Pleura and Peritoneum; PIPAC, Pressurized intraperitoneal aerosol chemotherapy; PIPAC-CD, PIPAC with cisplatin and doxorubicin; PIPAC-OX, PIPAC with oxaliplatin; PM, Peritoneal metastases; PRGS, Peritoneal regression grading score.

* Corresponding author. Department of Surgical Oncology, Montpellier Cancer Institute, 208, Avenue des Apothicaires, 34298, Montpellier, France,

E-mail address: Olivia.sgarbura@icm.unicancer.fr (O. Sgarbura).

\section{Introduction}

Pressurized intraperitoneal aerosol chemotherapy (PIPAC) has been developed as a treatment alternative for patients with various forms of peritoneal cancer [1,2]. Initial evaluation by the German pioneer center was followed by adoption of a handful of academic centers in Europe with high degree of standardization of the mostly empirical treatment protocol [3]. Due to excellent tolerance and safety profile as well as encouraging preliminary reports [4], interest was growing rapidly and a structured training curriculum was 
elaborated by clinical leaders of active training centers under the umbrella of the International Society of the Pleura and Peritoneum (ISSPP) [5] as a mandatory instruction before clinical practice. A very recently updated systemic review confirmed encouraging oncological outcomes but also pinpointed the exponential growth of PIPAC use all over the world and outside academic institutions [6]. The use of other types of intraperitoneal chemotherapy, especially hyperthermic intraperitoneal chemotherapy (HIPEC), has known not only tremendous clinical growth but also a high variability of applied protocols in terms of duration, drug choice, temperature [7] leading to difficult pooling of their data.

There are hence issues of concern with regards to potential deviation from the current standardized PIPAC protocol which might induce safety questions but also undermine the credibility of multi-center evaluation of PIPAC treatment which is now the next mandatory step according to the IDEAL framework of surgical innovation $[6,8,9]$.

The aim of the present study was to assess current PIPAC practice in terms of technique, treatment and safety protocols, and indications in all available international centers.

\section{Methods}

A standardized survey on PIPAC practice was elaborated by the faculty members $(\mathrm{n}=5)$ during a one-day kick-off meeting before the ISSPP PIPAC training workshop in Montpellier in September 2019. The survey contains 82 questions (mostly closed-ended multiple-choice) on center demographics, surgical technique, safety aspects, details on drug regimens, administration of PIPAC, treatment protocols, indications and contraindications, and the PIPAC registry (online appendix). The items covered all crucial aspects of an efficacious and safe PIPAC treatment in a concise survey taking a median of $8 \mathrm{~min}$ (tested by the core team). Of note, no patient-sensible information was included in this survey, and ethical clearance was hence not necessary.

The target population for this practice survey consisted of all the active PIPAC centers. PIPAC training centers and local distributors of the PIPAC-specific nebulizer were contacted to obtain names and email addresses of the leading physicians of active PIPAC centers. Cross-checks were performed by screening authors/centers of PubMed-listed publications on PIPAC. No center was voluntarily excluded from this study.

The survey was formatted to fit to SurveyMonkey (SVMK Inc, San Mateo, CA), and sent online one by one via personal invitations highlighting purpose and importance of this inquiry. Non-responders were re-contacted twice, again by personal contact. The survey was closed 7 weeks after initial launch.

\section{Statistics and analysis}

Plain descriptive statistics were used to present the results of current PIPAC practice. Percentages were rounded to the nearest integer number. A comparison between experienced ( $>60$ cumulative PIPACs performed) and novice centers was thought to be of interest. For this purpose, variables were reported as median and range. Categorical variables were compared using Chi-square test and continuous variables using Mann Whitney test. Correlations were scrutinized using Pearson's test. A two-sided alpha of 0.05 was used to indicate statistical significance. The statistical analysis was performed using Stata 16 software (StataCorp LLC, College Station, Texas, USA).

\section{Results}

\section{Participating centers}

Sixty-six active PIPAC centers were identified and 62 of them answered the survey giving a response rate of $93 \%$. A total number of declared 5972 PIPACs procedures had been performed by all participating centers after removal of duplicates. The identified centers came from 20 countries (Fig. 1). The median number of procedures per patient was 5.5 among centers (range 2-18). The median number of cumulative performed PIPAC was 41 (range $1-999)$. Two institutions had exceptionally high volumes ( $>500$ cases) while 25 institutions were considered high-volume (more than 60 PIPACs performed). Thirteen institutions were private centers. The median number of surgeons performing PIPAC in each center was two (range 1-10). Fifty-nine centers (95\%) had a program dedicated to peritoneal metastases (PM) and 57 centers $(92 \%)$ also performed HIPEC in eligible cases. Three institutions started the program before 2015, eleven joined in 2015 and the diffusion of the technique continued with 8 centers in 2016, 12 in 2017, 13 in 2018, and 15 in 2019 respectively.

All inquired aspects on current PIPAC practice are described in the supplementary material (online appendix). Some of the details will be highlighted by category of data.

\section{Surgical technique}

The PIPAC technique was frequently based on the use of two trocars but two teams used the single-port access. Systemic use of balloon trocars was declared by 54 responders (87\%). Thirty-tree

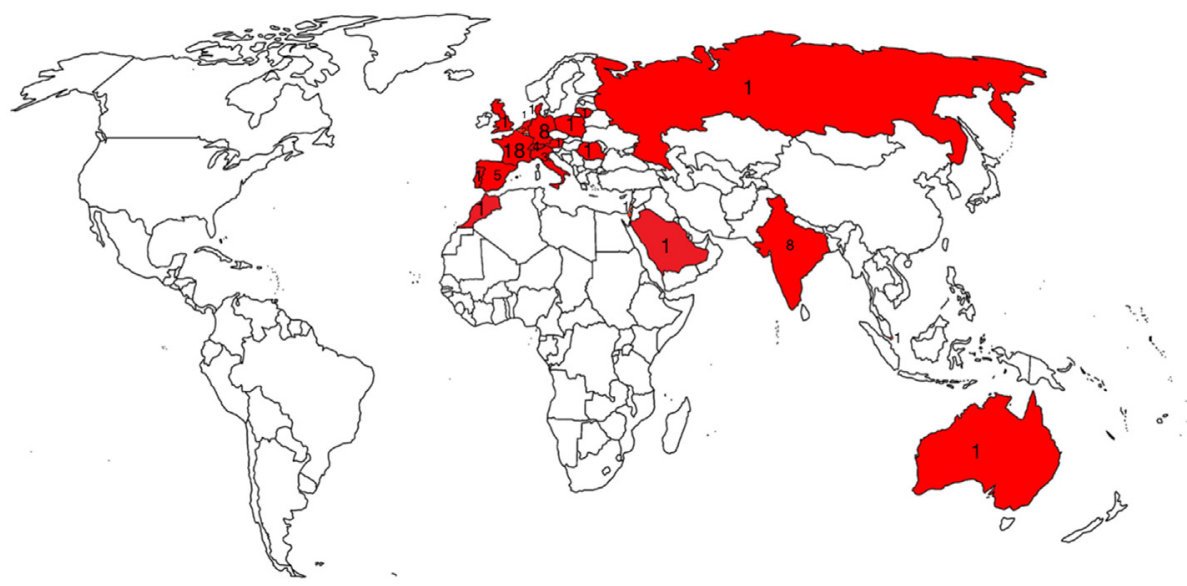

Fig. 1. Geographical distribution of the participating centers. 
teams (53\%) preferred the midline positioning of the trocars and 50 teams $(80 \%)$ always inserted the first trocar under the Hasson technique. US-guided trocar insertion was used by 4 centers (6\%). In case of repeated PIPAC, 54 (87\%) centers favored the use of the same trocar sites.

\section{Safety protocol}

Thirty-fourth (55\%) centers used the validated German safety protocol while 26 (42\%) opted for the French safety protocol. PIPAC teams used advanced air ventilation systems (laminar or other). Eleven centers (17\%) did not comply with advanced ventilation system requirements. The safety checklist was systematically used before PIPAC administration by 95\%. Limited adhesiolysis appeared to be acceptable for 39 participants (63\%); in case of accidental bowel injury, 52 centers (84\%) would defer PIPAC administration. Acceptance for concomitant surgical procedures is displayed in Fig. 2.

\section{Response evaluation}

During PIPAC, different modalities for assessing response to treatment were available and they were submitted to the respondents. Global consensus on response evaluation was $59.6 \%$. The different answers are reproduced in Table 1. Peritoneal Regression Grading Score (PRGS) was used by most teams (81\%). Peritoneal cytology was systematically performed in $34 \%$ of cases.

\section{PIPAC protocol}

The temperature of the cytotoxic drugs (room temperature, no heating) and the pressure settings (working pressure $12 \mathrm{mmHg}$, maximal upstream pressure 290psi/20 bar) were similar among
Table 1

Evaluation of the disease response during PIPAC cycles.

\begin{tabular}{lll}
\hline PCI evaluation & Systematically & $59(95 \%)$ \\
& Sometimes & $2(3 \%)$ \\
Biopsies & Missing answers & $1(2 \%)$ \\
& Median number (range) & $3(1-4)$ \\
& 3 biopsies & $29(47 \%)$ \\
& 4 biopsies & $21(34 \%)$ \\
& Not a fixed number & $11(17 \%)$ \\
Normal peritoneum & Missing answers & $1(2 \%)$ \\
& Systematically & $16(26 \%)$ \\
& Occasionally & $25(40 \%)$ \\
Biopsies in cases of repeated procedure & Never & $20(32 \%)$ \\
& Missing answers & $1(2 \%)$ \\
& Same sites & $17(27 \%)$ \\
Pathological assessment & Alternate sites & $44(71 \%)$ \\
& Missing answers & $1(2 \%)$ \\
& PRGS & $50(81 \%)$ \\
& No & $8(13 \%)$ \\
Peritoneal cytology & Other scores & $3(4 \%)$ \\
& Missing answers & $1(2 \%)$ \\
& Systematically & $21(34 \%)$ \\
& Only if ascites & $31(50 \%)$ \\
& Never & $9(14)$ \\
& Missing & $1(2)$ \\
\hline
\end{tabular}

responders (online appendix), but flow rate and administration time were different (Fig. 3). The use of ePIPAC was recorded in 9 centers but under different durations and sequences (online appendix).

\section{Chemotherapy regimens}

Cisplatin-doxorubicin association (PIPAC CD) was used by 57 (92\%) centers while oxaliplatin was used by 46 centers (74\%). The preferred dose for PIPAC CD was cisplatin $7.5 \mathrm{mg} / \mathrm{m}^{2}$ and doxorubicin

Incisional hernia repair or parastomial hernia without mesh

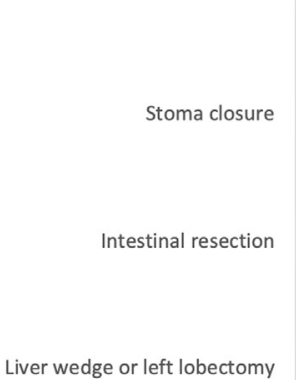

Adnexectomy

Appendicectomy

Appendicectom

Cholecystectomy
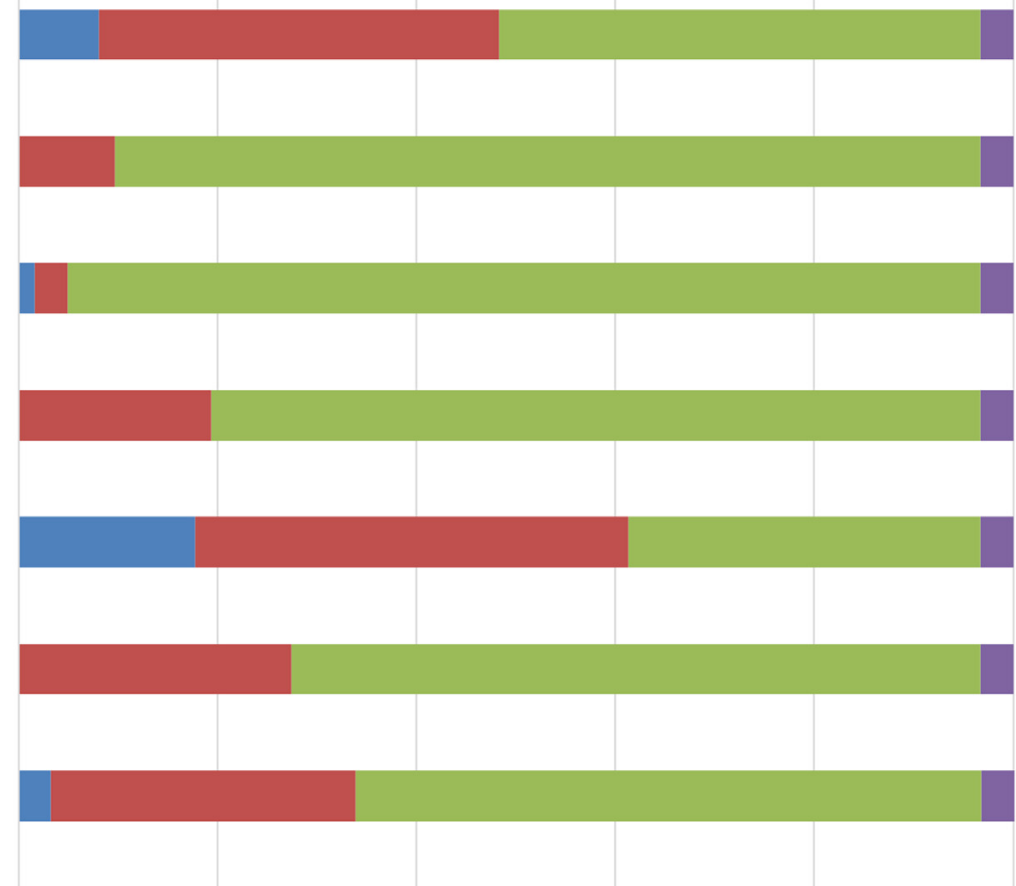

Omentectomy

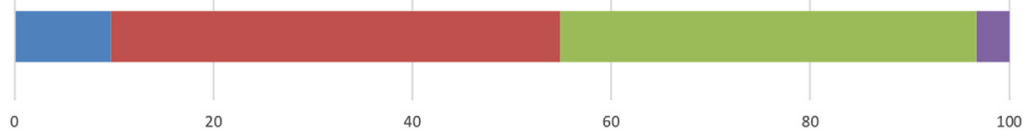

Done already $\quad$ Not done but acceptable $\quad$ should not be done missing

Fig. 2. Acceptability of concomitant procedures during PIPAC. 


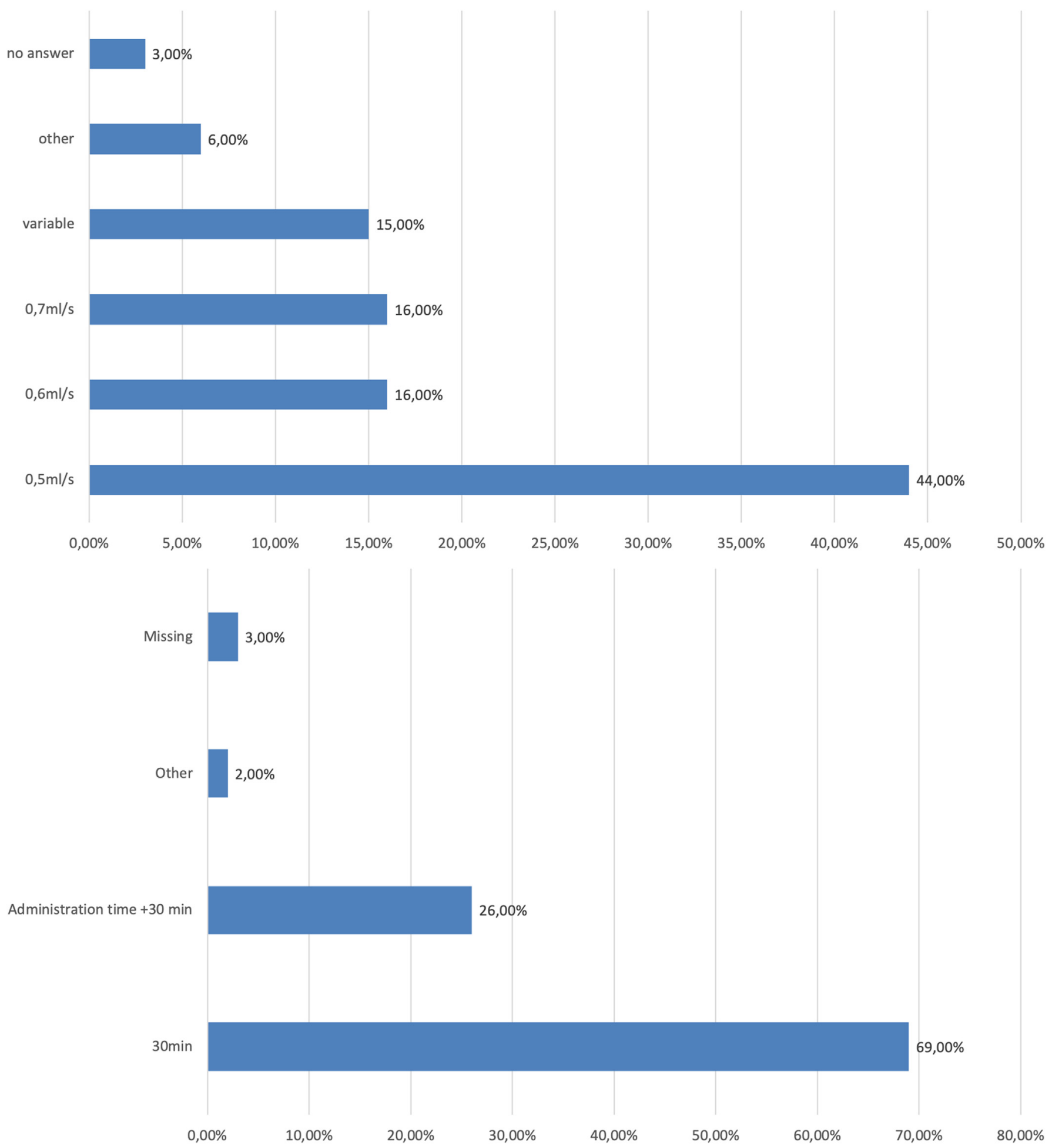

Fig. 3. Flow rate (a) and administration time (b) distribution.

$1.5 \mathrm{mg} / \mathrm{m}^{2}$ in 33 centers (53\%) and the phase-I recommended dose (cisplatin $10.5 \mathrm{mg} / \mathrm{m}^{2}$ and doxorubicin $2.1 \mathrm{mg} / \mathrm{m}^{2}$ ) in 24 centers (35\%), respectively. For oxaliplatin-based PIPAC (PIPAC Ox), 8 centers (13\%) used concomitant 5FU. When PIPAC CD was used, cisplatin was the first injected drug for 40 teams (64.5\%). Mitomycin C at $1.5 \mathrm{mg} /$ $\mathrm{m}^{2}$ was used by 6 centers $(10 \%)$ and irinotecan $\left(20 \mathrm{mg} / \mathrm{m}^{2}\right)$ by one single center. Thirty-nine centers (63\%) started with an intended number of 3 PIPACs, and continue thereafter either with 3 supplementary cycles $(n=21,35 \%)$ or one by one $(n=39,63 \%)$. The delay between systemic chemotherapy and PIPAC as well as potential association with anti-VEGF are presented in the online appendix.

\section{Indications for PIPAC}

Indications for PIPAC according to active PIPAC centers are displayed for the 6 main oncological entities in Fig. 4, according to the clinical setting (palliative treatment, neoadjuvant/induction treatment, adjuvant treatment, maintenance treatment, prophylactic 


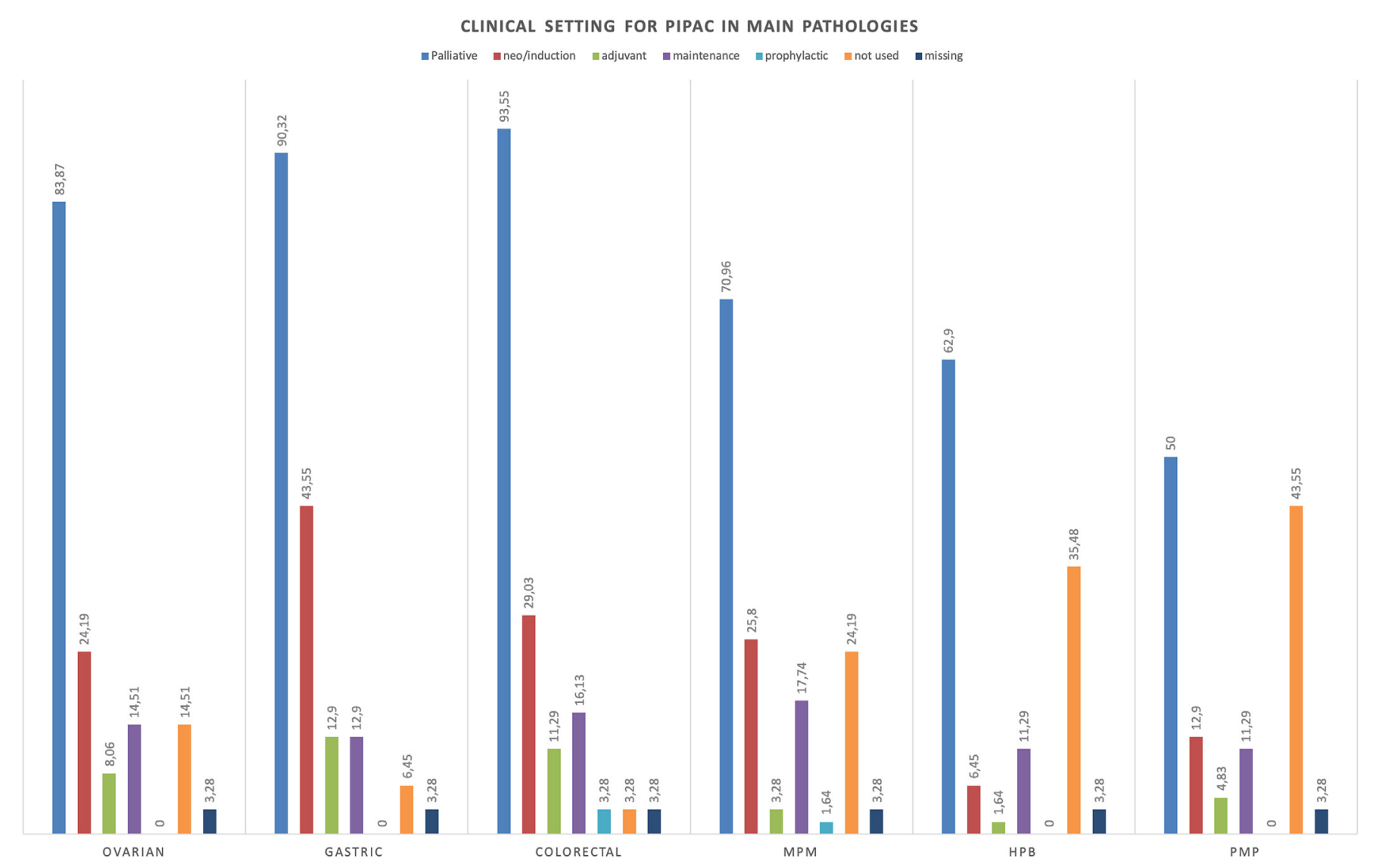

Fig. 4. PIPAC indications according to pathologic entity and clinical setting.

administration). The respondents were also questioned about the recent changes in practice in the last 6 months before the survey. Indications (40\%) and flow-rate (16\%) were the most frequently cited changes.

\section{Registry}

Forty-nine centers (79\%) attached a high importance to having a PIPAC registry, but only 16 centers (26\%) contributed into the previous registry. Main hurdles were time restraints (58\%), unresolved regulation issues (40\%), and costs (27\%).

\section{Analysis of homogeneity}

After exclusion of the open-end items and of the demographical items, 79 closed-end items were to be answered. Consensus of more than $80 \%$ of the centers was encountered for 28 items (35\%). Consensus of more than $70 \%$ of the centers was encountered for 37 items (47\%). Median consensus rate was $69.5 \%$ and their variation by item is presented in Fig. 5. When median consensus rates were compared among them, there was no statistical difference. When the percentages were compared, the safety consensus rate was significantly higher than the lowest two categories (chemotherapy and evaluation of the response) with $\mathrm{p}<0.01$. When the lack of adhesion to the items with $80 \%$ consensus was considered, it did not correlate with the type of institution (public versus private), with the date of the PIPAC program start, nor with the volume of the institution $(\mathrm{p}>0.05)$.

\section{Discussion}

The multiplication of protocols for locoregional administration of chemotherapy based on dose or duration variations is one of the dangers that deeply affected HIPEC [7] and led to the present survey concerning the practice of PIPAC. It was conducted in all identified active centers worldwide and demonstrated that the PIPAC procedure is performed with a uniform concern for safety but with an increasing variability related to surgical aspects, chemotherapy regimens and response evaluation.

The current practice assessment was performed two years after the initial survey based on 832 procedures in 349 patients [3]. By the present time, more than 1800 procedures were systematically reviewed in the literature [6] and some centers accumulated as many as 1200 procedures [10]. The current results are based on a declared cumulative experience of the interviewed centers of 5927 procedures which is significantly towering compared to the previous reports.

The response rate was high (93\%). A moderately high consensus (>70\%) was identified for $47 \%$ of all items with a median consensus rate of $69.5 \%$. These results indicate a rather good standardization of this technique.

Nevertheless, we identified an increasing variability compared to the previous situation in regards that might be crucial to safety or efficacy. Whereas consensus on safety measures was significantly higher than in all other aspects, we still pointed out isolated situations where the checklist was not applied by either new-coming teams or experimented teams formed by a variety of surgeons. The almost equal distribution of safety protocols among French and German models is related to the validating course [5] followed by each team and to the national regulations. Thus, it is not a ground 


\section{Median consensus rate}

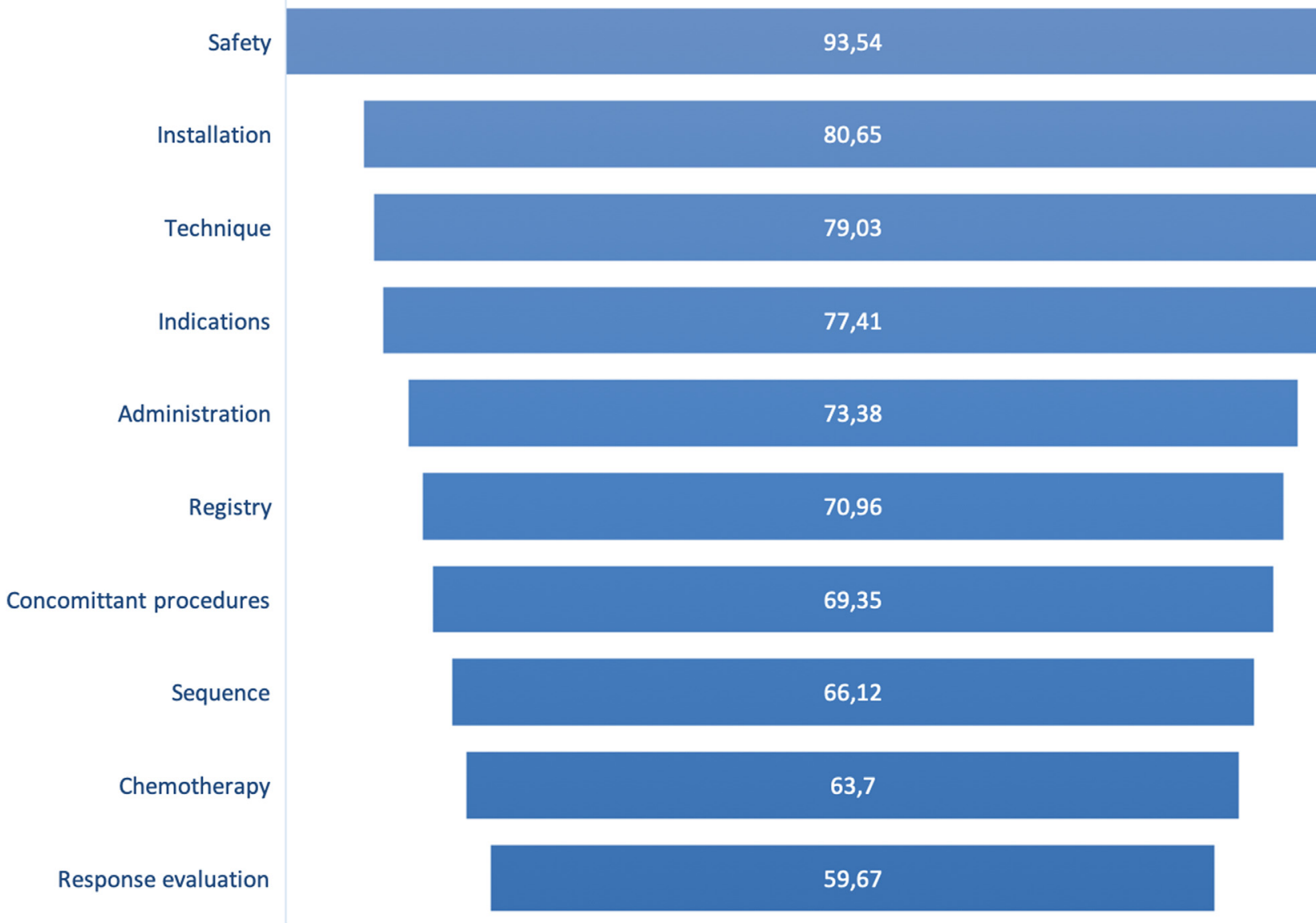

Fig. 5. Variation of median consensus rates by tested category.

for heterogeneity as both protocols have proved their efficacy [11-13]. The same considerations apply regarding institutional preferences for installation, access [14-16], closure and size of the camera as they probably have no implications on outcome.

However discrepancies were noted concerning the use of chemotherapy, doses for PIPAC-Ox and PIPAC-CD, sequence planning and response evaluation. Initial drug associations for PIPAC were empirical $[1,17]$ but Phase I studies concerning the phase-2 recommended dose were already published for PIPAC CD [18] and are ongoing for PIPAC-Ox [19,20] and Abraxane [21]. However, the current survey showed that the higher doses for PIPAC CD are almost as frequently applied as the initial doses. A potential explanation would be the lack of systemic chemotherapy in combination with PIPAC in the phase I study which favours good tolerance to the local drug administration. As some authors report association between PIPAC-CD and systemic chemotherapy [22] for non-ovarian malignancies, it is not yet known whether the phase I dose is as well tolerated in a combination treatment as in monotherapy. New technologies such as ePIPAC $[23,24]$ or currently under-study molecules [25] are expected to increase variations as well while other factors such as flow-rate or pressure are currently empirically changed based on manufacturer recommendations but in the absence of reliable experimental data.

A PIPAC expert Delphi consensus based on the current literature could address these issues in order to prevent further diversification of protocols in the absence of new evidence. Preservation of a standardized treatment protocol allows pooling of the data resulting in higher quality scientific evidence concerning PIPAC in the waiting of randomized controlled studies [26-28].

Evaluation of the treatment response for peritoneal metastases is challenging as several types of radiological examinations are needed to improve PCI estimation [29]. Laparoscopic evaluation was showed to underestimate the extent of PCI in colorectal PM [30] and $\delta \mathrm{PCI}$ was recently identified as in independent prognostic factor for OS after cytoreductive surgery and HIPEC [31], although it may still represent a subjective measurement. PRGS was recently proposed and proved to be reproducible [32]. Even with limited evidence about its prognostic role [33], PRGS represents one of the directions of research concerning the response evaluation explaining the large proportion of centers (81\%) routinely performing it. However, the fact that none of the current tools (radiology, laparoscopic PCI, $\delta$ PCI, PRGS) are strongly validated for PM of different origins was reflected in the low global rate of consensus (59.6\%) concerning modalities of response assessment.

The declared cumulative experience of the centers involved in this study ( $\mathrm{n}=5927$ procedures) is an indicator of the difference between the clinical numbers and the literature numbers for this procedure. Although the IDEAL evaluation for this technology emphasized the fact that many teams published their safety data (IDEAL stage 0 and 1) [8], based on these results we can infer that presently clinical data is available for large cohorts of patients and efficacy studies (IDEAL stage $2 \mathrm{~b}$ ).

This analysis reported self-declared practice. In theory, the actual situation in the respective centers might be different, variable (multiple users) or might have experienced change over time. In practice, PIPAC is delivered in most centers at this early stage by 
one programme leader with clear institutional standard. Despite all efforts, probably not all active PIPAC centers have been reached by the survey. The authors are confident however, to have contacted the large majority of them covering a close to total number of performed PIPACs worldwide [6]. The high response rate of 93\% should hence give a representative picture of current PIPAC practice.

In summary, homogeneous treatment standards are important especially for new techniques to allow for safe implementation and clinical practice but also to allow comparison between cohorts and multi-center analysis of merged data including registries. The present study raised concerns about increasing diversification of PIPAC practice. Ongoing efforts aiming for standardization include regular update of the PIPAC training curriculum, targeted research and a consensus statement.

\section{Disclosures}

None.

\section{CRediT authorship contribution statement}

Olivia Sgarbura: Conceptualization, Methodology, Validation, Formal analysis, Data curation, Writing - original draft, Writing review \& editing. Laurent Villeneuve: Validation, Formal analysis, Data curation, Writing - original draft, Writing - review \& editing. Mohammad Alyami: Conceptualization, Methodology, Validation, Data curation, Writing - original draft, Writing - review \& editing. Naoual Bakrin: Conceptualization, Methodology, Validation, Writing - original draft, Writing - review \& editing. Juan José Torrent: Methodology, Validation, Writing - original draft, Writing - review \& editing. Clarisse Eveno: Validation, Formal analysis, Data curation, Writing - original draft, Writing - review \& editing, Conceptualization, Methodology. Martin Hübner: Conceptualization, Methodology, Validation, Formal analysis, Data curation, Writing - original draft, Writing - review \& editing.

\section{Declaration of competing interest}

No conflicts of interest.

\section{Acknowledgements}

The authors would like to than Mrs Caroline Mollevi for statistical advice.

\section{Appendix A. Supplementary data}

Supplementary data to this article can be found online at https://doi.org/10.1016/j.ejso.2020.08.020.

\section{References}

[1] Reymond MA, Giger-Pabst U, Solass W, Tannapfel A, Strumberg D, Zieren J. First clinical experience with pressurized intraperitoneal aerosol chemotherapy in patients with gastric cancer and peritoneal carcinomatosis. Surg Endosc Other Interv Tech 2014. https://doi.org/10.1007/ s00464-014-3483-0.

[2] Solass W, Kerb R, Mürdter T, Giger-Pabst U, Strumberg D, Tempfer C, et al. Intraperitoneal chemotherapy of peritoneal carcinomatosis using pressurized aerosol as an alternative to liquid solution: first evidence for efficacy. Ann Surg Oncol 2014;21:553-9. https://doi.org/10.1245/s10434-013-3213-1.

[3] Nowacki M, Alyami M, Villeneuve L, Mercier F, Hubner M, Willaert W, et al. Multicenter comprehensive methodological and technical analysis of 832 pressurized intraperitoneal aerosol chemotherapy (PIPAC) interventions performed in 349 patients for peritoneal carcinomatosis treatment: An international survey study. Eur J Surg Oncol 2018. https://doi.org/10.1016/ j.ejso.2018.02.014.
[4] Grass F, Vuagniaux A, Teixeira-Farinha H, Lehmann K, Demartines N, Hübner M. Systematic review of pressurized intraperitoneal aerosol chemotherapy for the treatment of advanced peritoneal carcinomatosis. Br J Surg 2017. https://doi.org/10.1002/bjs.10521.

[5] Alyami M, Sgarbura O, Khomyakov V, Horvath P, Vizzielli G, So J, et al. Standardizing training for pressurized intraperitoneal aerosol chemotherapy. Eur J Surg Oncol 2020. https://doi.org/10.1016/j.ejso.2020.05.007.

[6] Alyami M, Hübner M, Grass F, Bakrin N, Villeneuve L, Laplace N, et al. Pressurised intraperitoneal aerosol chemotherapy: Rationale, evidence, and potential indications. Lancet Oncol 2019;20:e368-77. https://doi.org/10.1016/ S1470-2045(19)30318-3.

[7] Yurttas C, Hoffmann G, Tolios A, Haen SP, Schwab M, Königsrainer I, et al. Systematic review of variations in hyperthermic intraperitoneal chemotherapy (HIPEC) for peritoneal metastasis from colorectal cancer. J Clin Med 2018. https://doi.org/10.3390/jcm7120567.

[8] Tate SJ, Torkington J. Pressurized intraperitoneal aerosol chemotherapy: a review of the introduction of a new surgical technology using the IDEAL framework. BJS Open 2020;4:206-15. https://doi.org/10.1002/bjs5.50257.

[9] McCulloch P, Altman DG, Campbell WB, Flum DR, Glasziou P, Marshall JC, et al. No surgical innovation without evaluation: the IDEAL recommendations. Lancet 2009;374:1105-12. https://doi.org/10.1016/S0140-6736(09)61116-8.

[10] Giger-Pabst U, Tempfer CB. How to perform safe and technically optimized pressurized intraperitoneal aerosol chemotherapy (PIPAC): Experience after a consecutive series of 1200 procedures. J Gastrointest Surg 2018;22:2187-93. https://doi.org/10.1007/s11605-018-3916-5.

[11] Ndaw S, Hanser O, Kenepekian V, Vidal M, Melczer M, Remy A, et al. Occupational exposure to platinum drugs during intraperitoneal chemotherapy. Biomonitoring and surface contamination. Toxicol Lett 2018;298:171-6. https://doi.org/10.1016/j.toxlet.2018.05.031.

[12] Ametsbichler P, Böhlandt A, Nowak D, Schierl R. Occupational exposure to cisplatin/oxaliplatin during pressurized intraperitoneal aerosol chemotherapy (PIPAC)? Eur J Surg Oncol 2018;44:1793-9. https://doi.org/10.1016/ j.ejso.2018.05.020.

[13] Solaß W, Giger-Pabst U, Zieren J, Reymond MA. Pressurized intraperitoneal aerosol chemotherapy (PIPAC): occupational health and safety aspects. Ann Surg Oncol 2013. https://doi.org/10.1245/s10434-013-3039-x.

[14] Vaira M, Robella M, Borsano A, De Simone M. Single-port access for pressurized IntraPeritoneal aerosol chemotherapy (PIPAC): Technique, feasibility and safety. Pleura and Peritoneum 2016. https://doi.org/10.1515/pap-2016-0021.

[15] Glatz T, Horvath P, Lang SA, Archid R, Nadiradze G. Staging laparoscopy and Pressurized IntraPeritoneal Aerosol Chemotherapy (PIPAC) for peritoneal metastasis: Safe access to the abdomen. Pleura and Peritoneum 2019;4. https://doi.org/10.1515/pp-2019-0004.

[16] Hübner M, Grass F, Teixeira-Farinha H, Pache B, Mathevet P, Demartines N. Pressurized IntraPeritoneal aerosol chemotherapy - practical aspects. Eur J Surg Oncol 2017;43:1102-9. https://doi.org/10.1016/j.ejso.2017.03.019.

[17] Sgarbura O, Hubner M, Alyami M, Eveno C, Gagnière J, Pache B, et al. Oxaliplatin use in pressurized intraperitoneal aerosole chemotherapy(PIPAC) is safe and well tolerated: A multicenter study. Eur J Surg Oncol 2019;45:e60. https://doi.org/10.1016/j.ejso.2018.10.226.

[18] Tempfer CB, Giger-Pabst U, Seebacher V, Petersen M, Dogan A, Rezniczek GA. A phase I, single-arm, open-label, dose escalation study of intraperitoneal cisplatin and doxorubicin in patients with recurrent ovarian cancer and peritoneal carcinomatosis. Gynecol Oncol 2018. https://doi.org/10.1016/ j.ygyno.2018.05.001.

[19] Dumont F, Senellart H, Pein F, Campion L, Glehen O, Goere Di, et al. Phase I/II study of oxaliplatin dose escalation via a laparoscopic approach using pressurized aerosol intraperitoneal chemotherapy (PIPOX trial) for nonresectable peritoneal metastases of digestive cancers (stomach, small bowel and colorectal): Rationale. Pleura and Peritoneum 2018. https://doi.org/10.1515/pp2018-0120.

[20] Kim G, Tan HL, Chen E, Teo SC, Jang CJM, Ho J, et al. Study protocol: Phase 1 dose escalating study of pressurized intraperitoneal aerosol chemotherapy (PIPAC) with oxaliplatin in peritoneal metastasis. Pleura and Peritoneum 2018. https://doi.org/10.1515/pp-2018-0118.

[21] Van De Sande L, Graversen M, Hubner M, Pocard M, Reymond M, Vaira M, et al. Intraperitoneal aerosolization of albumin-stabilized paclitaxel nanoparticles (Abraxane ${ }^{\mathrm{TM}}$ ) for peritoneal carcinomatosis - a phase I first-inhuman study. Pleura and Peritoneum 2018;3. https://doi.org/10.1515/pp2018-0112.

[22] Alyami M, Gagniere J, Sgarbura O, Cabelguenne D, Villeneuve L, Pezet D, et al. Multicentric initial experience with the use of the pressurized intraperitoneal aerosol chemotherapy (PIPAC) in the management of unresectable peritoneal carcinomatosis. Eur J Surg Oncol 2017;43:2178-83. https://doi.org/10.1016/ j.ejso.2017.09.010.

[23] Graversen M, Detlefsen S, Ellebaek SB, Fristrup C, Pfeiffer P, Mortensen MB. Pressurized IntraPeritoneal Aerosol Chemotherapy with one minute of electrostatic precipitation (ePIPAC) is feasible, but the histological tumor response in peritoneal metastasis is insufficient. Eur J Surg Oncol 2019. https://doi.org/ 10.1016/j.ejso.2019.08.024.

[24] Willaert W, Van de Sande L, Van Daele E, Van De Putte D, Van Nieuwenhove Y, Pattyn P, et al. Safety and preliminary efficacy of electrostatic precipitation during pressurized intraperitoneal aerosol chemotherapy (PIPAC) for unresectable carcinomatosis. Eur J Surg Oncol 2019. https://doi.org/10.1016/ j.ejso.2019.06.018. 
[25] Tan H, Kim G, Jang C, Shabbir A, Charles C, Li R, et al. PIPAC paclitaxel: A systematic and peritoneal tissue pharmacokinetic study in swine. Ann Oncol Off J Eur Soc Med Oncol 2019. https://doi.org/10.1093/annonc/mdz155.268.

[26] Bakrin N, Tempfer C, Scambia G, De Simone M, Gabriel B, Grischke EM, et al PIPAC-OV3: a multicenter, open-label, randomized, two-arm phase III trial of the effect on progression-free survival of cisplatin and doxorubicin as Pressurized Intraperitoneal Chemotherapy (PIPAC) vs. Chemotherapy alone in patients with platinum-resistan. Pleura and Peritoneum 2018. https://doi.org/ 10.1515/pp-2018-0114.

[27] Eveno C, Jouvin I, Pocard M. PIPAC EstoK 01: pressurized IntraPeritoneal Aerosol Chemotherapy with cisplatin and doxorubicin (PIPAC C/D) in gastric peritoneal metastasis: A randomized and multicenter phase II study. Pleura and Peritoneum 2018. https://doi.org/10.1515/pp-2018-0116.

[28] Sgarbura O, Gourgou S, Tosi D, Bakrin N, Bouazza N, Delaine S, et al. MESOTIP: Phase II multicenter randomized trial evaluating the association of PIPAC and systemic chemotherapy vs. systemic chemotherapy alone as 1st-line treatment of malignant peritoneal mesothelioma. Pleura and Peritoneum 2019;4. https://doi.org/10.1515/pp-2019-0010.

[29] Dohan A, Hoeffel C, Soyer P, Jannot AS, Valette PJ, Thivolet A, et al. Evaluation of the peritoneal carcinomatosis index with CT and MRI. Br J Surg 2017 https://doi.org/10.1002/bjs.10527.

[30] Passot G, Dumont F, Goéré D, Arvieux C, Rousset P, Regimbeau JM, et al. Multicentre study of laparoscopic or open assessment of the peritoneal cance index (BIG-RENAPE). Br J Surg 2018. https://doi.org/10.1002/bjs.10723.

[31] Hentzen J, van der Plas W, Kuipers H, Ramcharan S, Been L, Hoogwater F et al. Delta Peritoneal Cancer Index $(\triangle \mathrm{PCI})$ : a new dynamic prognostic parameter for survival in patients with peritoneal carcinomatosis from colorectal cancer. Eur J Surg Oncol 2019;45:e57-8. https://doi.org/10.1016/ j.ejso.2018.10.220.

[32] Solass W, Sempoux C, Carr NJ, Bibeau F, Neureiter D, Jäger T, et al. Reproducibility of the peritoneal regression grading score for assessment of response to therapy in peritoneal metastasis. Histopathology 2019;74: 1014-24. https://doi.org/10.1111/his.13829.

[33] Benzerdjeb N, Durieux E, Tantot J, Isaac S, Fontaine J, Harou O, et al. Prognostic impact of combined progression index based on peritoneal grading regression score and peritoneal cytology in peritoneal metastasis. Histopathology 2020. https://doi.org/10.1111/his.14092. his.14092. 